\title{
Gradual Change Reliability Sensitivity Design for Components with Arbitrary Distribution Parameters Based on Measured Information
}

\author{
Xingang Wang ${ }^{1}$, Baoyan Wang ${ }^{1, *}$, Miaoxin Chang $^{2}$ and Mingming Yan ${ }^{2}$ \\ ${ }^{1}$ Center of Mechanical Reliability \& Dynamics, Northeastern University, Qinhuangdao, 066004, China \\ ${ }^{2}$ School of Mechatronics Engineering, Harbin Institute of Technology, Harbin, Heilongjiang 150001, China \\ ${ }^{*}$ Corresponding author
}

\begin{abstract}
Reliability design of mechanical components mostly focused on establishing pure theoretical mathematical model at present, without involving measured information for working components and gradual characteristics of parameters into theoretical model, which caused some error for reliability design of components. In order to access reliability of existing components correctly, by taking strength of components as a process of independent increments, autocorrelation coefficient of strength is calculated, and effect of loading action and gradual change characteristics of strength is studied, thus a method for computing gradual change reliability is proposed. Combining the reliability design theory with sensitivity analysis method, a numerical method for gradual change reliability sensitivity design of components based on measured information is proposed, and the variation rules of reliability sensitivity of parameters at any moment and effects of design parameters on reliability of components are obtained, which provides the theoretical basis for structural design and life prediction of mechanical components.
\end{abstract}

Keywords-strength of materials; gradual change reliability; sensitivity analysis; stochastic process models; arbitrary distribution parameters; reliability design

\section{INTRODUCTION}

At present, a lot of research on mechanical gradual change reliability have been did by many scholars, yet most of which is to set up mathematical models of pure theory, without involving measured information for working components and gradual characteristics of parameters into theoretical model, so some errors is brought into the reliability design. Although gradual change characteristics of loads are studied deeply, gradual change characteristics of strength still need further research ${ }^{[1]}$. It's mainly because there are lots of factors causing the strength degeneration of components, coupled with the change of physical properties of the material itself, it is hard to show the non-stationary stochastic process of strength degeneration using a certain mathematical model. In this paper, certain work was done about this problem. We fully used the measured value of components at current moment to make correction about the hypothetical stochastic process model of strength ${ }^{[2]}$. Here, the hypothetical model is regarded to be failure, and using the measured values to amend it. Thus we can get the model on stochastic process of strength for existing components in the future using period.

\section{Gradual Change Performance of Stress}

For mechanical structure and components in existence, the fact that strength degenerate and random loads change with time due to the influence of external work environment and internal factors cannot be neglected. Formula of gradual change reliability is represented as follows,

$$
R(t)=\Phi(\beta(t))=P\{r(t)>\sigma(t) \quad t \in[0, T]\}
$$

The formula (1) shows that when the strength is greater than load at each moment of its design service period, components' structure is in the reliable state.

In order to identify the influence of equivalent maximum load of this random load on components, the random load is required to be equally discreted as $\mathrm{n}$ parts from the moment ti to a certain moment ti+ $\triangle \mathrm{t}$. If structure is not failure under the action of the maximum load Smax of $n$ parts, components structure can't be in failure under the action of $n$ times of random load. That is,

$$
\begin{aligned}
& P(t)=P\left\{r>\sigma\left(S_{\max }\right)\right\}= \\
& P\left\{r>\sigma\left(S_{1}\right), r>\sigma\left(S_{2}\right), \cdots, r>\sigma\left(S_{n}\right)\right\}
\end{aligned}
$$

where $\sigma(\mathrm{Si})$ is stress response caused by the load $\mathrm{Si}$.

Therefore, reliability after the effect of $\mathrm{n}$ times of random load is equivalent to the corresponding reliability of maximum value of $n$ load samples. The maximum load in the load samples can be defined as the equivalent load of $n$ times' load effect. According to order statistic theory, the maximum load Smax actually is the maximum of order statistic variable Sn. From extremum distribution principle of the maximum item, distribution function of the maximum load effect $\sigma(\operatorname{Smax})$ in the continuous $n$ equal intervals, can be given as follows[3]

$$
\begin{aligned}
& F_{\tau}(x)=P\left(\max _{1 \leq i \leq n} S_{i} \leq x\right)= \\
& P\left(S_{1} \leq x\right) P\left(S_{2} \leq x\right) \cdots P\left(S_{n} \leq x\right)=\prod_{i=1}^{n} P\left(S_{i} \leq x\right)=\left[F_{\tau}(x)\right]^{n}
\end{aligned}
$$


Thus a model of reliability of load is set up. The formula of reliability under the stochastic action of load is given as,

$$
R(t)=P\left\{r>\max \sigma(t) \quad t \in\left[t_{i}, t_{i}+\Delta t\right]\right\}
$$

where max $\sigma(t)$ is the maximum equivalent load effect of load's stochastic process.

\section{Gradual Change Performance of Strength}

Because the great uncertainty of correlation among each strength at any moment exists, in this paper, using independent increment principle of stochastic process, we view correlation among each strength as independent increment of stochastic process, and make corrections to the original stochastic process model of strength according to the measured value at current moment, thus a stochastic process model of strength degeneration for components in future working time is formed. Then suppose stochastic process of strength degeneration to be $\left\{r(t), \mathrm{t} \in\left[t_{\mathrm{i}}, t_{\mathrm{i}}+\Delta t\right]\right\}$, strength $r\left(t_{\mathrm{i}}\right)$ is a random variable at the present moment $t_{\mathrm{i}}$, its mean value is $E\left[r\left(t_{\mathrm{i}}\right)\right]$, variance is $D\left[r\left(t_{\mathrm{i}}\right)\right]$. The original stochastic process of strength degeneration is $\left\{r_{0}(t), t \in\left[t_{\mathrm{i}}, t_{\mathrm{i}}+\Delta t\right]\right\}$, and its mean value function is $E\left[r_{0}(t)\right]$, variance function is $D\left[r_{0}(t)\right] . E\left[r\left(t_{\mathrm{i}}\right)\right]$ is equal to $E\left[r_{0}\left(t_{\mathrm{i}}\right)\right], D\left[r\left(t_{\mathrm{i}}\right)\right]$ is equal to $D\left[r_{0}\left(t_{\mathrm{i}}\right)\right]$ at current moment $t_{\mathrm{i}}$ in the meaning of statistics and theory. Then the stochastic process of strength is defined as ${ }^{[4]}$

$$
r(t)=r\left(t_{i}\right)+\left[r_{0}(t)-r_{0}\left(t_{i}\right)\right]
$$

The mean value function and the variance function are given as follows

$$
\begin{aligned}
& E[r(t)]=E\left[r\left(t_{i}\right)\right]+E\left[r_{0}(t)\right]-E\left[r_{0}\left(t_{i}\right)\right] \\
& D[r(t)]=D\left[r\left(t_{i}\right)\right]+D\left[r_{0}(t)\right]+D\left[r_{0}\left(t_{i}\right)\right]- \\
& 2 \operatorname{cov}\left[r_{0}(t), r_{0}\left(t_{i}\right)\right]
\end{aligned}
$$

where $\operatorname{cov}[\mathrm{r} 0(\mathrm{t}), \mathrm{r} 0(\mathrm{ti})]$ is the covariance function of strength degeneration. If $\{\mathrm{r}(\mathrm{t}), \mathrm{t} \in[\mathrm{ti}, \mathrm{ti}+\triangle \mathrm{t}]\}$ is independent increment process, the autocorrelation coefficient of strength is

$$
\rho_{r}\left[r\left(t_{i}\right), r\left(t_{i}+\Delta t\right)\right]=\frac{\operatorname{cov}\left[r\left(t_{i}\right), r\left(t_{i}+\Delta t\right)\right]}{\sqrt{D\left[r\left(t_{i}\right)\right] D\left[r\left(t_{i}+\Delta t\right)\right]}}
$$

It can be proved by the property of the independent increment process as follows,

$$
\rho_{r}\left[r\left(t_{i}\right), r\left(t_{i}+\Delta t\right)\right]=\sqrt{\frac{D\left[r\left(t_{i}\right)\right]}{D\left[r\left(t_{i}+\Delta t\right)\right]}}
$$

By substituting equation (8-9) into equation (7), the variance function of strength degeneration is obtained

$$
D[r(t)]=D\left[r\left(t_{i}\right)\right]+D\left[r_{0}(t)\right]-D\left[r_{0}\left(t_{i}\right)\right]
$$

From the measured sample value at current moment $t_{\mathrm{i}}$, the formulas of mean value, variance and autocorrelation coefficient of strength in the mechanical parts' future service period $t \in\left[t_{\mathrm{i}}, t_{\mathrm{i}}+\triangle t\right]$ can be ascertained by $(6-10)$.

\section{The Design on Gradual Change Reliability}

\section{A. Calculation of Dynamic Reliability Index}

According to formula (1), the structural reliability of components is expressed by function equation as follows,

$$
R(t)=P\{g(t)>0, t \in[0, T]\}
$$

The formula (11) is to show that when $r(t)$ at each moment $t$ during a period of design service is greater than $\sigma(t)$, component structure is in the reliable state. The probability of failure is

$$
F(t)=1-R(t)=P\{r(t)<\sigma(t), t \in[0, T]\}
$$

Considering measured value of components at current time $t_{\mathrm{i}}$, reliability in the mechanical parts' future service period $\left[t_{\mathrm{i}}\right.$, $\left.t_{\mathrm{i}}+\Delta t\right]$ can be given as

$$
R(t)=P\left\{r\left(t_{i}\right)-\sigma\left(t_{i}\right)>0, t_{i} \in\left[t_{i}, t_{i}+\Delta t\right]\right\}
$$

where $r\left(t_{i}\right)$ is the strength of components at $t_{\mathrm{i}}$ moment; $\sigma\left(t_{\mathrm{i}}\right)$ is effect of random load at any moment $t_{\mathrm{i}}$. According to formula (13), a component is equal to a series system made up by $n$ samples in the period of whole design service. Basis on definition of reliability of the series system, in order to make the whole system effective, every subsystem must be effective too. And the gradual change reliability is

$$
R(t)=P\left\{\bigcap_{i=1}^{n} g\left(t_{i}\right)>0, t_{i} \in\left[t_{i}, t_{i}+\Delta t\right]\right\}
$$

Suppose $\beta(\mathrm{ti})$ to be $\Phi-1\{\mathrm{~g}(\mathrm{ti})>0, \mathrm{i}=1,2, \ldots, \mathrm{n}\}, \rho \mathrm{T}$ is the correlation coefficient matrix among state functions within every equal time interval, the formula is $\rho \mathrm{T}=\rho \mathrm{g}[\mathrm{g}(\mathrm{ti}), \mathrm{g}(\mathrm{tj})] \mathrm{n} \times \mathrm{n}$; $\Phi n(\cdot, \cdot)$ is standard normal distribution function of $\mathrm{n}$ dimensions, $\mathrm{nn}-1(\cdot, \cdot)$ is inverse function of $\Phi \mathrm{n}(\cdot, \cdot)$. On the basis of the independent increment process, correlation coefficient of the state function is 


$$
\begin{aligned}
& \rho_{g}\left[g\left(t_{i}\right), g\left(t_{j}\right)\right]=\frac{\operatorname{cov}\left[g\left(t_{i}\right), g\left(t_{j}\right)\right]}{\sqrt{D\left[r\left(t_{i}\right)\right] D\left[r\left(t_{j}\right)\right]}}= \\
& \frac{\rho_{r}\left[r\left(t_{i}\right), r\left(t_{j}\right)\right] \sqrt{D\left[r\left(t_{i}\right)\right] D\left[r\left(t_{j}\right)\right]}}{\sqrt{\left\{D\left[r\left(t_{i}\right)\right]+D\left[\sigma\left(t_{i}\right)\right]\right\}\left\{D\left[r\left(t_{j}\right)\right]+D\left[\sigma\left(t_{j}\right)\right]\right\}}}
\end{aligned}
$$

$$
(i \neq j, i=1,2, \ldots, \mathrm{n} ; j=1,2, \ldots, n)
$$

The reliability of components

$$
R(t)=\int_{-\infty}^{+\infty} \varphi(t) \prod_{i=1}^{n}\left\{\frac{1}{\sqrt{2 \pi}} \int_{-\infty}^{\beta^{\prime}\left(t_{i}\right)} \exp \left(-\frac{u^{2}}{2}\right) d u\right\} d t
$$

\section{B. Random Perturbation Method}

The vector $\boldsymbol{X}$ of random parameters and its state function $g(\boldsymbol{X})$ are related with time parameter $t$, so they are expanded as $^{[5]}$

$$
\begin{gathered}
\mathbf{X}(t)=\mathbf{X}(t)_{d}+\varepsilon \mathbf{X}(t)_{p} \\
g(\mathbf{X}, t)=g_{d}(\mathbf{X}, t)+\varepsilon g_{p}(\mathbf{X}, t)
\end{gathered}
$$

where $\varepsilon$ is a small parameter. Parts of equations (17) and (18) expressed by subscript $d$ are certain parts of the random parameters, and parts expressed by subscript $\mathrm{p}$ are the random parts, having a zero mean value in the random parameters.

By expanding function $g_{p}(X, t)$ to first-order approximation at a point $\mathrm{E}(\boldsymbol{X}(\mathrm{t}))=\boldsymbol{X}(\mathrm{t})_{\mathrm{d}}$ according to Taylor series of vector value and matrix functions, which is on the failure surface $\mathrm{g}_{\mathrm{p}}\left(\boldsymbol{X}(\mathrm{t})_{\mathrm{d}}\right)=0$, the expression of $\mathrm{g}_{\mathrm{p}}(\boldsymbol{X}, \mathrm{t})$ is given

$$
g_{p}(\boldsymbol{X}, t)=\frac{\partial g_{d}(\boldsymbol{X}, t)}{\partial \boldsymbol{X}(t)^{\mathrm{T}}} \boldsymbol{X}(t)_{p}
$$

After knowing the first four moments, that is to say mean value, variance, covariance, the third moment and the fourth moment of the basic random parameter $X$, reliability index can be defined as

$$
\beta\left(t_{i}\right)=\frac{3\left(\alpha_{4 g_{i}}-1\right) \beta\left(t_{i}\right)+\alpha_{3 g_{i}}\left(\beta\left(t_{i}\right)^{2}-1\right)}{\sqrt{\left(5 \alpha_{3 g_{i}}^{2}-9 \alpha_{4 g_{i}}+9\right)\left(1-\alpha_{4 g_{i}}\right)}}
$$

Substituting equation (20) into equation (16), the new reliability index with arbitrary distribution parameter can be given as

$$
\beta\left(t_{i}\right)=\frac{3\left(\alpha_{1 g_{i}}-1\right) \beta\left(t_{i}\right)+\alpha_{3 g_{i}}\left(\beta\left(t_{i}\right)^{2}-1\right)}{\sqrt{\left(5 o_{3 g_{i}}^{2}-9 \alpha_{1 g_{i}}+9\right)\left(1-\alpha_{1 g_{i}}\right)\left(1-\rho_{g_{i}}\right)}}-\sqrt{\frac{\rho_{g_{i}}}{1-\rho_{g_{i}}}} t
$$

In engineering practice, the available data may be sufficient to evaluate the first few moments such as mean, variance, the third moment, and the fourth moment of the random variables. Based on the first four moments of original random variables, the reliability index can be obtained by using the perturbation method. For a state function of unknown probability distribution, it is approximately expressed as standard normal distribution by the first four moments by using the Edgeworth expansion. Thus, reliability and the reliability sensitivity of mechanical components with non-normal distribution parameters can be obtained.

\section{Calculation of Gradual Change Reliability Sensitivity}

As reliability index $\beta$ and the random variable vector are related with time, the reliability sensitivity with respect to the mean value of the original random parameter vector $\boldsymbol{X}$ is derived as

$$
\begin{gathered}
\frac{\mathrm{D} R(t)}{\mathrm{D}(\overline{\mathbf{X}}, t)^{\mathrm{T}}}=\frac{\partial R\left(\beta^{\prime}(t)\right)}{\partial \beta^{\prime}(t)} \frac{\partial \beta^{\prime}(t)}{\partial \beta(t)} \frac{\partial \beta(t)}{\partial \mu_{g(t)}} \frac{\partial \mu_{g(t)}}{\partial(\overline{\mathbf{X}}, t)^{\mathrm{T}}}+ \\
{\left[\frac{\partial R\left(\beta^{\prime}(t)\right)}{\partial \beta^{\prime}(t)} \frac{\partial \beta^{\prime}(t)}{\partial \beta(t)} \frac{\partial \beta(t)}{\partial \sigma_{g(t)}}+\frac{\partial R\left(\beta^{\prime}(t)\right)}{\partial \sigma_{g(t)}}\right] \frac{\partial \sigma_{g(t)}}{\partial(\overline{\mathbf{X}}, t)^{\mathrm{T}}}}
\end{gathered}
$$

where $I_{q}$ and $U_{q^{\times} q}$ denote unit matrix of $q \times q$ dimension and a matrix of $q^{2} \times q^{2}$ dimension respectively.

\section{NUMERICAL EXAMPLE}

\section{A. Mechanical Model of Rear-axle Housing}

An axle casing is both force transmission and loading component (in Figure 1.). With the high-speed and lightweight development of special vehicle in modern military field, rearaxle housing composed by steel stamping, steel tubes expansion and forging appeared.

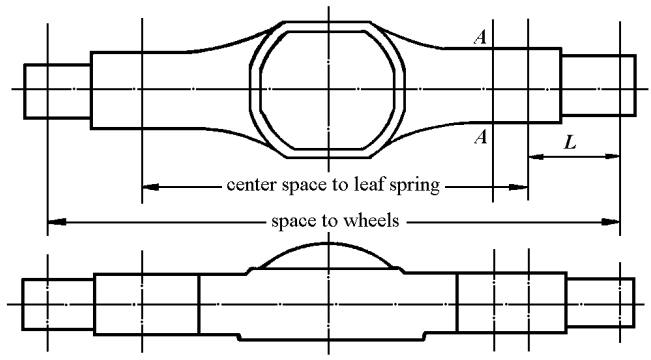

FIGURE I. STRUCTURE OF REAR-AXLE HOUSING

The rear-axle housing is generally loaded torsional moment and bending moment. The risk section is on two sides of leaf spring seats and fillet of a flange. For some kinds of vehicle, 
section is quadrate with a circular hole. Regarding quadrate section with a circular hole, the rear-axle housing effected mainly bending stress are defined as

$$
\sigma=\frac{M}{W_{n}}
$$

where $M$ is bending moment. $W_{n}$ is the sectional coefficient, which is represented as

$$
W_{n}=\frac{b h^{2}}{6}\left(1-0.59 \frac{d^{4}}{b h^{3}}\right)
$$

According to expression (1), the state equation of rear-axle housing is defined as

$$
g(s)=r(t)-\frac{M(s)}{W_{n}}
$$

\section{B. Computation of the Model}

For rear-axle housing of sixty-ton transporter, which has been under forty thousands $\mathrm{km}$ service, its risk section is quadrate with a circular hole. Mean value and standard deviation $d=(110,0.6) \mathrm{mm}$ of inside diameter of section. $b$ and $h$ are side length of quadrate section, and mean and standard deviation are $b=(130,0.8) \mathrm{mm}$ and $h=(156,0.68) \mathrm{mm}$ respectively. The measured mean function and variance function of its strength are $E[r(s)]=1.7 \mathrm{e}^{-0.00006 s}$ and $D[r(s)]=4.2 \exp \left(-3 \times 10^{-8} s^{2}\right)$ respectively; The loaded torsional moment of rear-axle housing with the first four moments is $M=\left(1.1 \times 10^{7} \mathrm{~N} \cdot \mathrm{mm}, 9.7 \times 10^{6}\right.$ $\mathrm{N} \cdot \mathrm{mm}, 265.3 \times 10^{6} \mathrm{~N} \cdot \mathrm{mm}^{3}, 125.6 \times 10^{8} \mathrm{~N} \cdot \mathrm{mm}^{4}$ ) at the current moment, and the maximum equivalent load effect of load stochastic process obeys extremum distribution.

From figure 2, it is accord with the actual working condition that structural reliability $R$ of the rear-axle housing is gradually reduced with the increase of vehicle operation. Figure 3 to 5 show changes of sensitivity of parameters $b, h$ and $d$, it can be seen that reliability $R$ with respect to sensitivity of $h$ is larger than that of $b$ and $d$ at any time, thus $h$ is the most sensitive. Mean value of $b$ and $h$ is the larger, and the rear-axle housing is more reliable. Sensitivity of inside diameter $d$ is negative, which shows the rear-axle housing tends to be unreliable, that failure rate becomes larger with the increase of mean value of $d$.

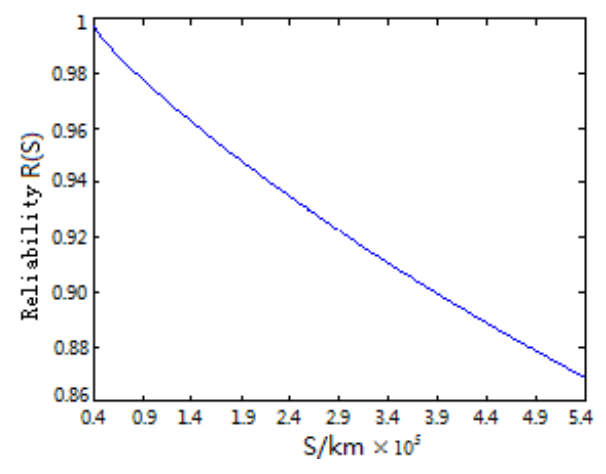

FIGURE II. CURVE OF TIME-DEPENDENT RELIABILITY OF LAND AXLE HOUSING.

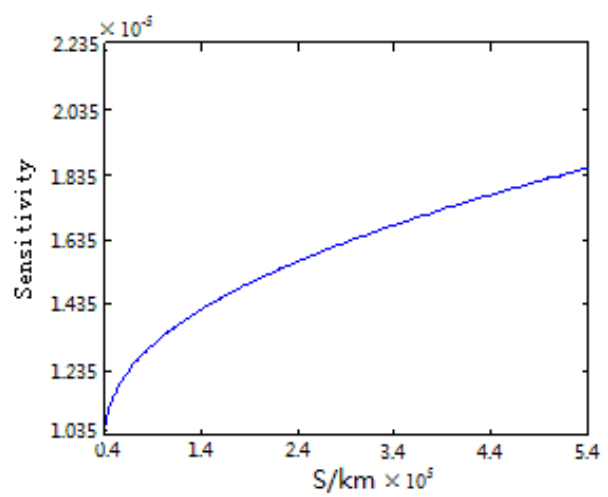

FIGURE III. CURVE OF RELIABILITY SENSITIVITY OF $B$

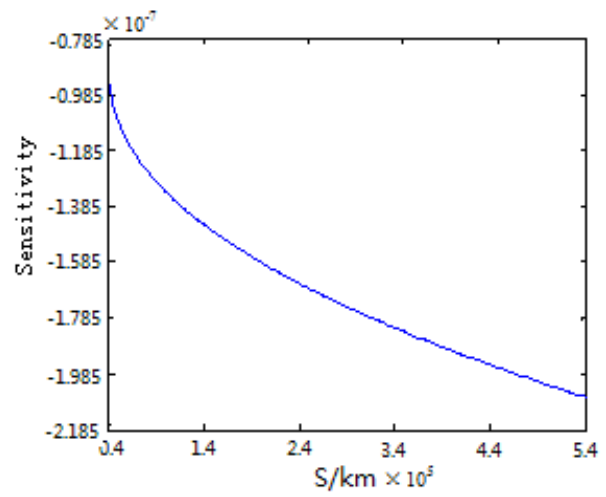

FIGURE IV. CURVE OF RELIABILITY SENSITIVITY OF $D$

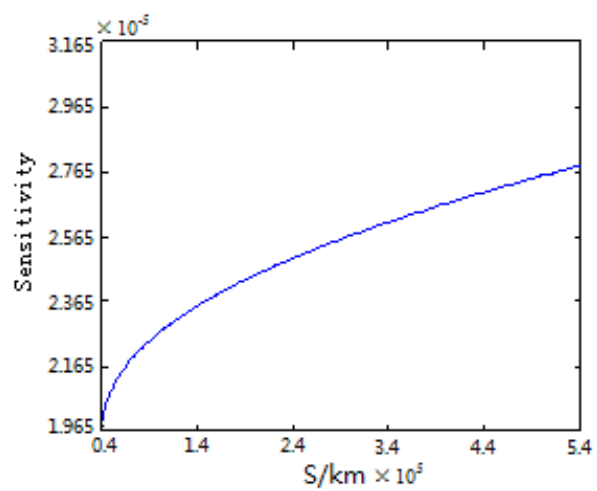

FIGURE V. CURVE OF RELIABILITY SENSITIVITY OF $H$ 


\section{CONCLUSIONS}

(1) This paper makes full use of measured information of current moment to modify original stochastic process model of strength, and an innovative model of strength is obtained for existing components in future using time, thus we can evaluate reliability of components correctly.

(2) Distribution types of each parameter can be relaxed in this paper, even though parameters obey any distribution or distributions are unknown at all, we can still make design on reliability and sensitivity of components.

\section{ACKNOWLEDGMENT}

Project supported by the National Natural Science Foundation of China (Grant No. 51475086); supported by "the Fundamental Research Funds for the Central Universities (Grant No. N152304004); Project Supported by the Natural Science Foundation of Liaoning province of China (Grant No. 2014020026); Project Supported by the Natural Science Foundation of Hebei province of China (Grant No. E2015501073)

\section{REFERENCES}

[1] Faggioni M, Samani S F, Bertacchi G, et al. Dynamic optimization of spur gears[J]. Mechanism and Machine Theory,2010,46(4):544-557.

[2] Bonori G, Barbieri M, Pellicano F. Optimum profile modifications of spur gears by means of genetic algorithms[J]. Jourrral of Sourrd and Vibration, 2008, 313 (3/4/5):603-616.

[3] WANG XINGANG, ZHANG YIMIN, WANG BAOYAN. Reliabilitybased design of automobile components[J]. Proceedings of the Institution of Mechanical Engineers, Part C, Journal of Mechanical Engineering Science.2009,223(C2),483-490.

[4] ZHANG JUNZHI. The Dynamic Reliability of Proof Load for Existing Structures[J]. JOURNAL OF WUHAN UNIVERSITY OF TECHNOLOGY, 2000,22(6):75-77.

[5] ZHANG YIMIN. Reliability Design of Automobile Components[M]. Beijing, Beijing Institute of Technology Press, 2000 